\title{
Hysteresis Loops, Critical Fields and Energy Products for Exchange-spring Hard/soft/hard Trilayers
}

\author{
B. Z. Chen ${ }^{1}$, S. Yan ${ }^{1}$, Y. Z. Ju' ${ }^{1}$ G. P. Zhao ${ }^{2 *}$, X. C. Zhang' ${ }^{2}$, M. Yue ${ }^{3 *}$, and J. Xia ${ }^{2}$ \\ ${ }^{1}$ Traffic and Transportation School, Dalian Jiaotong University, Dalian 116028, China \\ ${ }^{2}$ College of Physics and Electronic Engineering, Sichuan Normal University, Chengdu 610068, China \\ ${ }^{3}$ College of Materials Science and Engineering, Beijing University of Technology, Beijing 100124, China
}

(Received 1 April 2014, Received in final form 26 February 2015, Accepted 10 March 2015)

\begin{abstract}
Macroscopic hysteresis loops and microscopic magnetic moment distributions have been determined by a three-dimensional (3D) model for exchange-coupled $\mathrm{Sm}-\mathrm{Co} / \mathrm{\alpha}-\mathrm{Fe} / \mathrm{Sm}-\mathrm{Co}$ trilayers with in-plane collinear easy axes. These results are carefully compared with the popular one-dimensional (1D) micromagnetic models and recent experimental data. It is found that the results obtained from the two methods match very well, especially for the remanence and coercivity, justifying the calculations. Both nucleation and coercive fields decrease monotonically as the soft layer thickness $L^{s}$ increases while the largest maximum energy product (roughly 50 MGOe) occurs when the thicknesses of hard and soft layers are $5 \mathrm{~nm}$ and $15 \mathrm{~nm}$, respectively. Moreover, the calculated angular distributions in the thickness direction for the magnetic moments are similar. Nevertheless, the calculated nucleation and pinning fields as well as the energy products by 3D OOMMF are systematically smaller than those given by the 1D model, due mainly to the stray fields at the corners of the films. These demagnetization fields help the magnetic moments at the corners to deviate from the previous saturation state and facilitate the nucleation. Such an effect enhances as $L^{s}$ increases. When the thicknesses of hard and soft layers are $10 \mathrm{~nm}$ and $20 \mathrm{~nm}$, respectively, the pinning field difference is as large as $30 \%$, while the nucleation fields have opposite signs.
\end{abstract}

Keywords : hard/soft trilayers, hysteresis loops, energy products, micromagnetic calculation

\section{Introduction}

The concept of exchange-springs, i.e., exchangecoupled hard/soft composite nanomagnets, was proposed by Kneller et al. in 1991 [1]. In a typical composite magnet, the magnetizations of the soft phase respond to the applied field reversibly and therefore these magnets are called exchange-springs [1-3]. Two years later, Skomski and Coey predicted that the energy product of exchangesprings can be as large as 120 MGOe [4], which doubles that of the sintered $\mathrm{Nd}_{2} \mathrm{Fe}_{14} \mathrm{~B}$, the best performed permanent magnets up to now. Many efforts have been devoted to realize such a giant energy product in the past two decades [2-9]. Nevertheless, the experimental energy products are much smaller, even smaller than the sintered $\mathrm{Nd}_{2} \mathrm{Fe}_{14} \mathrm{~B}$ magnets. Such a huge difference is called

CThe Korean Magnetics Society. All rights reserved.

*Corresponding author: Tel: +86-10-67391769

Fax: +86-10-67391769, e-mail: zhaogp@uestc.edu.cn,

yueming@bjut.edu.cn energy products in some literatures $[10,11]$. Therefore, a reexamination of the present theories regarding to the hysteresis loops and energy products in the exchange spring materials is necessary.

Skomski and Coey made their prediction based on a one dimensional (1D) micromagnetic model [4], which was first utilized by Goto et al. in 1960s [12]. Similar model has been widely used in the past two decades, by Leineweber and Kronmüller et al. from Germany [13], by Fullerton and Jiang et al. from USA [2, 14], by Asti and Pellicelli et al. from Italy [15-17] and by Zhao et al. from China $[10,11,18]$. Such a simple model can reveal some important underlying physics with analytical formulas derived. However, it can not take account of the magnetic distributions in the film plane as well as the sophisticated microstructures existed in nature.

Experimentally, a major progress has been made in the last year [9], where an energy product of 60 MGOe has been achieved in $\mathrm{NdFeB} / \mathrm{FeCo}$ multilayers by Cui et al. [9]. Such a high energy product surpasses that of the sintered $\mathrm{Nd}_{2} \mathrm{Fe}_{14} \mathrm{~B}$ already. In the same time, the energy 
products in SmCo-Fe system have aroused intense interests and made progress. SmCo is the hardest magnetic material in the world which can supply the large coercivity whilst the soft Fe can provide high remanence. As early as 1995, $\mathrm{SmCo} / \mathrm{FeCo}$ bilayers and multilayers have been fabricated by Al-Omari et al. [19]. where the room-temperature energy product is only 6 MGOe. In 2002, Cheng et al. [20] fabricated the $\mathrm{SmFeGaC} / \mathrm{Fe}$ permanent magnet, with the energy product increased to $8.2 \mathrm{MG}$ Oe. In 2005 , Neu et al. [21] realized an energy product of $28.13 \mathrm{MG}$ Oe $\left(224 \mathrm{~kJ} / \mathrm{m}^{3}\right)$ by improving the exchange coupling between $\mathrm{SmCo}$ and $\mathrm{Fe}$ layers. The $\mathrm{Fe} / \mathrm{Co}$ mixture behavior enhances the interface exchange-coupling and improves the remanence and coercivity of the composite magnet [22]. Similar work has been done by Choi et al. [23], where the largest energy product increases to $32 \mathrm{MG}$ Oe. The largest energy product for $\mathrm{Sm}-\mathrm{Co} / \mathrm{Fe}$ reported so far is around 40 MGOe, achieved by Sawatzki et al. [24] in $\mathrm{SmCo}_{5} / \mathrm{Fe} / \mathrm{SmCo}_{5}$ trilayers, where an interface $\mathrm{Fe}-\mathrm{Co}$ layer is formed which significantly improves the $(B H)_{\max }$. These superior energy products have been achieved with a well-oriented in-plane easy axis, as predicted by the present micromagnetic theory.

In this paper, the demagnetization process and hysteresis loops of $\mathrm{SmCo} / \mathrm{Fe} / \mathrm{SmCo}$ trilayer have been investigated using the 3D software (OOMMF) [25] and carefully compared with the results based on 1D analytic calculation.

\section{Micromagnetic Model}

The trilayer system studied in this paper is shown in Fig. 1, where a soft layer is sandwiched between two hard layers with collinear in-plane easy axes for both phases. A large magnetic field is applied along the easy axis direction and then gradually demagnetized to investigate the hysteresis loop related behaviors.

The three-dimensional (3D) calculation of the micromagnetic software package is based on the LandauLifshitz-Gilbert dynamic equation:

$$
\frac{d \mathbf{M}}{d t}=-|\bar{\gamma}| \mathbf{M} \times \mathbf{H}_{\mathrm{eff}}-\frac{|\bar{\gamma}| \alpha}{M_{S}} \mathbf{M} \times\left(\mathbf{M} \times \mathbf{H}_{\mathrm{eff}}\right),
$$

where $\mathbf{M}$ is the magnetization $\left(\mathrm{A} \cdot \mathrm{m}^{-1}\right), \mathbf{H}_{\mathrm{eff}}$ is the effective field $\left(\mathrm{A} \cdot \mathrm{m}^{-1}\right), \gamma$ is the Landau-Lifshitz gyromagnetic ration $\left(\mathrm{m} \cdot \mathrm{A}^{-1} \cdot \mathrm{s}^{-1}\right)$, and $\alpha$ is the dimensionless damping constant. The effective field is defined as follows:

$$
\mathbf{H}_{\text {eff }}=-\mu_{0}^{-1} \frac{\partial \mathrm{E}}{\partial \mathbf{M}} \text {. }
$$

The average energy density $E$ is a function of $\mathbf{M}$ specified by Brown's equations [26, 27]:

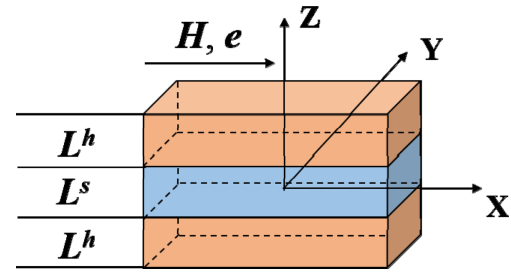

Fig. 1. (Color online) The hard-soft trilayer adopted for calculation in the present work.

$$
\begin{aligned}
\mathrm{E}= & A(r)\left[\frac{\nabla M}{M_{S}}\right]^{2}-K(r) \frac{(\mathbf{M} \cdot \mathbf{n})^{2}}{M_{S}}-\mu_{0} \mathbf{M} \cdot \mathbf{H} \\
& -\frac{1}{2} \mu_{0} \mathbf{H}_{\mathrm{d}}(r) \cdot \mathbf{M},
\end{aligned}
$$

where $A$ and $K$ are the exchange and anisotropy energy constants, respectively. $\mathbf{H}$ and $\mathbf{H}_{\mathrm{d}}(\mathrm{r})$ are the applied and magnetostatic self-interaction fields while $M_{S}=|\mathbf{M}(\mathrm{r})|$ is the spontaneous magnetization. These equations hold for both the hard and soft phases. The four terms at the $r$ ight side of Eq. (3) correspond to the exchange energy, the anisotropy energy, the applied field (Zeeman) energy and the magnetostatic (demagnetization) energy, respectively.

The above 3D energy could be simplified to a one dimensional (1D) expression if we ignore the magnetostatic interactions, which are small for an infinitely large thin film with in-plane easy axis. In one dimension, the energy density per area in the film plane is $[10,28]$ :

$$
\begin{aligned}
v= & \int_{0}^{L^{h}}\left[A^{h}\left(\frac{d \theta}{d z}\right)^{2}+K^{h} \sin ^{2} \theta-\mu_{0} H M_{s}^{h} \cos \theta\right] d z \\
& +\int_{-L^{h / 2}}^{0}\left[A^{s}\left(\frac{d \theta}{d z}\right)^{2}+K^{s} \sin ^{2} \theta-\mu_{0} H M_{s}^{s} \cos \theta\right] d z, \\
& -\frac{2 A^{h s}}{a}\left(\overrightarrow{m^{s}} \overrightarrow{m^{h}}-1\right)
\end{aligned}
$$

where $a$ is the distance between the adjacent atomic planes near the interface. $\theta$ is the angle between the magnetization and the applied field and $\boldsymbol{m}$ is the magnetization unit vector at the interface. For symmetry, only half of the trilayers has been included in the calculation. The three terms inside the bracket of the above formula are the exchange energy, the anisotropy energy and the Zeeman energy respectively, while the last term is the interface exchange coupling energy. A variational method $[10,28]$ is used to minimize the energy expressed in Eq. (4), which yields the equations for the angular distribution ( $\theta$ as a function of $z$ ) as follows?

For $0<z<L^{h}$ : 


$$
\frac{L^{h}-z}{\Delta^{h} / \pi}=\int_{\theta^{h}}^{\theta} \frac{d \theta}{\sqrt{\left(\sin ^{2} \theta-\sin ^{2} \theta^{h}\right)-2 h^{h}\left(\cos \theta-\cos \theta^{h}\right)}} .
$$

$$
\begin{aligned}
& \text { For }-L^{S} / 2<z<0: \\
& \frac{L^{s} / 2+z}{\Delta^{s} / \pi}=\int_{\theta}^{\theta^{s}} \frac{d \theta}{\sqrt{\left(\sin ^{2} \theta-\sin ^{2} \theta^{s}\right)-2 h^{s}\left(\cos \theta-\cos \theta^{s}\right)}} .
\end{aligned}
$$

where $h=H / H_{k}$ represents the reduced applied field with $H_{k}=2 K /\left(\mu_{0} M_{s}\right)$ as the anisotropy field. $\Delta=\pi \sqrt{A / K}$ is the Bloch wall width. $\theta^{h}$ and $\theta^{s}$ are the directions of the magnetization at the outer surfaces of the hard layer and the middle of the soft layer, respectively. From these equations the hysteresis loops and energy products can be calculated. The 1D calculation is basically an analytical one.

In this paper, Sm-Co is chosen as the hard layer while $\alpha-\mathrm{Fe}$ is the soft layer. The material parameters for the simulation are: $[2,29] M_{s}^{s}=1.7 \times 10^{3} \mathrm{emu} / \mathrm{cc}, M_{s}^{h}=0.55$ $\times 10^{3} \mathrm{emu} / \mathrm{cc}, K^{s}=1000 \mathrm{erg} / \mathrm{cc}, K^{h}=5 \times 10^{7} \mathrm{erg} / \mathrm{cc}, A^{s}=$ $2.8 \times 10^{-6} \mathrm{erg} / \mathrm{cm}, A^{h}=1.2 \times 10^{-6} \mathrm{erg} / \mathrm{cm}$, and $A^{h s}=1.8 \times$ $10^{-6} \mathrm{erg} / \mathrm{cm}$. $A, K$, and $M_{s}$ denote the exchange energy constant, the anisotropy constant, and the spontaneous magnetization, while the superscripts $h$ and $s$ refer to the hard and soft layers, respectively.

In the 3D simulation performed by OOMMF, the trilayer system is set as a tetragonal box, $300 \mathrm{~nm} \times 300$ $\mathrm{nm} \times L$, where $L$ is the thickness of the trilayer, which equals $2 L^{h}+L^{s}$ according to Fig. 1 . The thickness of the hard layer $L^{h}$ varies between $5 \mathrm{~nm}$ and $20 \mathrm{~nm}$, while that of the soft layer $L^{s}$ varies between $2 \mathrm{~nm}$ and $20 \mathrm{~nm}$. The system has been discretized into tetragonal cells with various sizes to find an optimum cell size where a good balance between the calculation precision and computational speed is obtained. The optimum cell size turns out to be $6 \mathrm{~nm} \times 6 \mathrm{~nm} \times 1 \mathrm{~nm}$, i.e., with both length and width set as $6 \mathrm{~nm}$ while keeping a fixed thickness of 1 $\mathrm{nm}$. The following calculations are based on such an optimum cell size unless otherwise specified. The applied field and the initial magnetization are along the $\mathrm{x}$ axis. Only the exchange interaction between the neighboring region pair is taken into account and the free boundary conditions are chosen.

\section{Macroscopic Hysteresis Loops}

Fig. 2 shows the macroscopic hysteresis loops of $\mathrm{SmCo} /$ $\mathrm{Fe} / \mathrm{SmCo}$ trilayers with various soft layer thickness and a fixed hard layer thickness of $10 \mathrm{~nm}$. The bottom and top panel correspond to the loops based on 3D and 1D methods respectively. The two methods give similar hysteresis

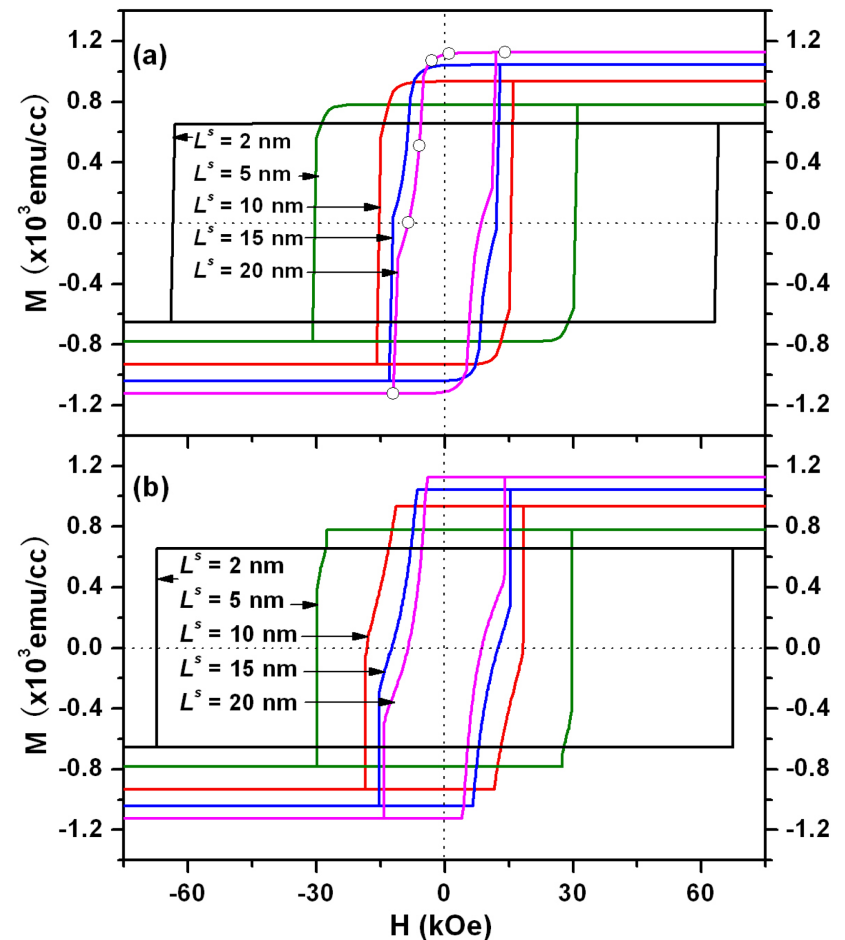

Fig. 2. (Color online) Calculated hysteresis loops for SmCo $(10 \mathrm{~nm}) / \mathrm{Fe}\left(L^{s}\right) / \mathrm{SmCo}(10 \mathrm{~nm})$ trilayers based on (a) OOMMF and (b) $1 \mathrm{D}$ analytical model. The hollow circles in the loop for $L^{s}=20 \mathrm{~nm}$ stand for some special states, which will be displayed in Fig. 4.

loops and very close coercive fields, justifying the calculations. When $L^{s}=2 \mathrm{~nm}$, both models yield rectangular hysteresis loops with roughly the same nucleation and pinning fields. As $L^{s}$ increases, the squareness of the hysteresis loops becomes worse, accompanied by the gradual decrease of the nucleation and coercive fields. Here, the nucleation field is defined as the opposite of the applied field at which the magnetization has some obvious deviations from the previous saturation state. In the meantime, the difference between the two calculated loops based on the two models enlarges. One can see that when $L^{s}=2 \mathrm{~nm}$, the calculated difference for the pinning field is within $6 \%$, which, however, increases to $30 \%$ as $L^{s}$ increases to $20 \mathrm{~nm}$. The pinning field is defined as the opposite of the applied field at which the magnetizations in the hard layer reverse, which is displayed in the hysteresis loops as a great drop of the magnetization. Further, the nucleation process calculated by the $3 \mathrm{D}$ simulation is smoother than the one based on the 1D method. In the 3D simulation, the stray fields at the corner of the film help the nucleation process and thus the magnetization reversal. These stray fields, neglected in the $1 \mathrm{D}$ model, smooth the nucleation process and decrease the nucleation fields. This phenomena can be seen more 


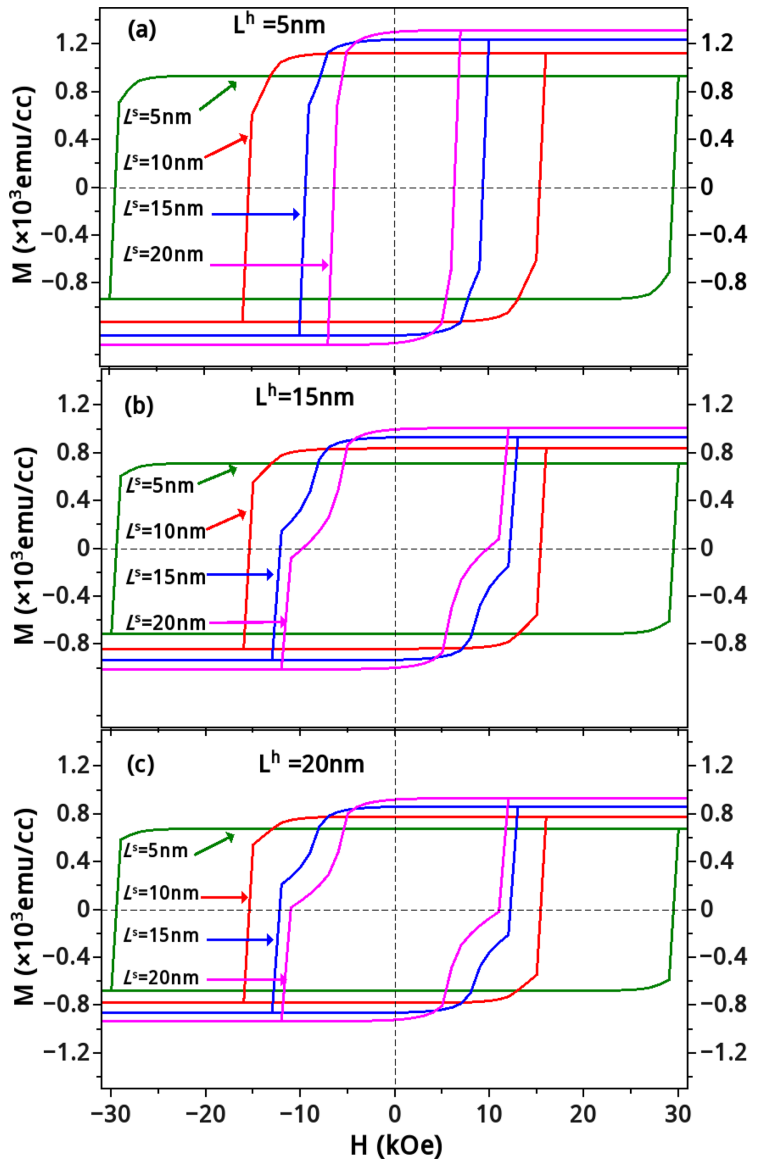

Fig. 3. (Color online) Calculated hysteresis loops for $\mathrm{SmCo}$ $\left(L^{h}\right) / \mathrm{Fe}\left(L^{s}\right) / \mathrm{SmCo}\left(L^{h}\right)$ trilayers based on OOMMF for various $L^{s}$, where $L^{h}=5 \mathrm{~nm}$ (top), $15 \mathrm{~nm}$ (middle) and $20 \mathrm{~nm}$ (bottom) respectively.

clearly from the in-plane magnetization distribution displayed in the next section.

Hysteresis loops have been calculated for SmCo $\left(L^{h}\right) /$ $\mathrm{Fe}\left(L^{s}\right) / \mathrm{SmCo}\left(L^{h}\right)$ trilayers based on OOMMF for other values of $L^{h}$ with various $L^{s}$ and shown in Fig. 3, where $L^{h}$ $=5 \mathrm{~nm}, 15 \mathrm{~nm}$ and $20 \mathrm{~nm}$ respectively. Similar to the case with $L^{h}=10 \mathrm{~nm}$. The squareness of the hysteresis loops is deteriorated as $L^{s}$ increases, accompanied by the gradual increase of the remanence and the decrease of both nucleation and coercive fields.

\section{Microscopic Magnetic Moment Distribution in the Film Plane}

Fig. 4 shows the $2 \mathrm{D}$ evolution of the magnetizations with the applied field for a $\operatorname{SmCo}(10 \mathrm{~nm}) / \mathrm{Fe}(20 \mathrm{~nm}) /$ $\mathrm{SmCo}(10 \mathrm{~nm})$ trilayer. These in-plane magnetic distributions calculated by the 3D OOMMF, are located at the middle of the soft layer $(z=0)$, which response to the applied field fast. At $H=14 \mathrm{kOe}$, the magnetizations at

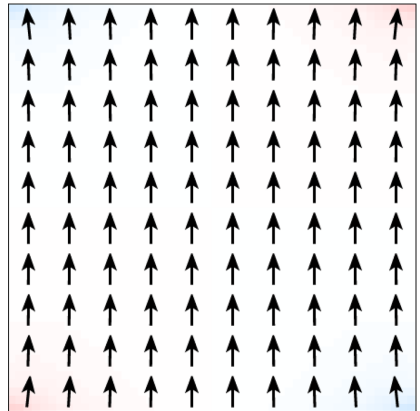

(a)

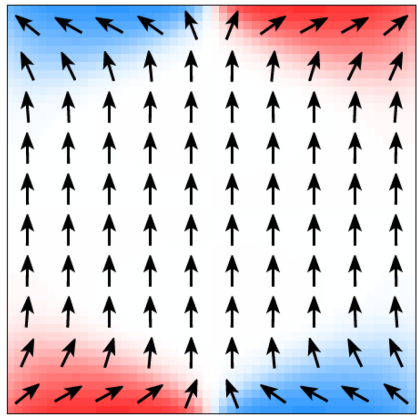

(c)

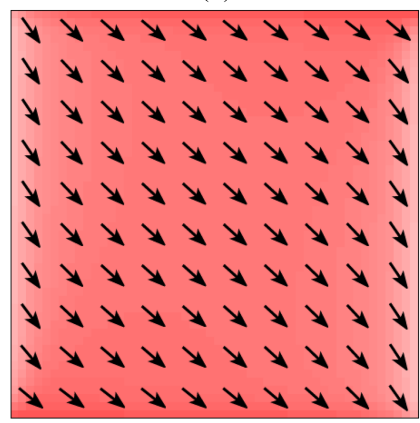

(e)

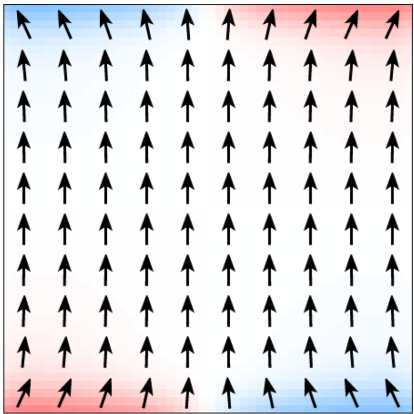

(b)

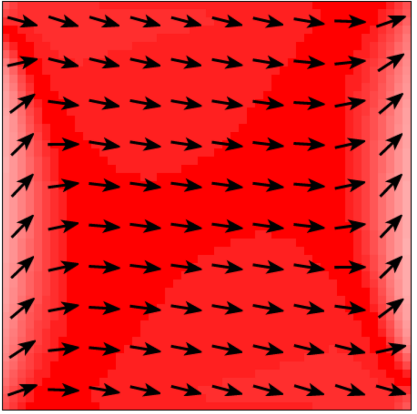

(d)

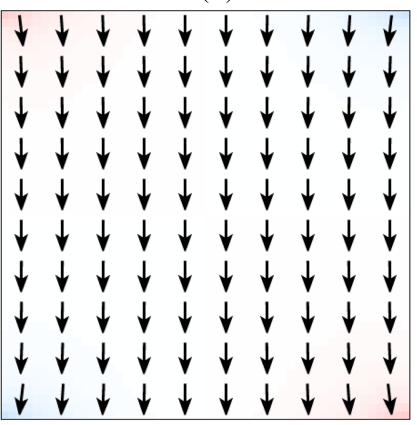

(f)
Fig. 4. (Color online) 2D evolution of the magnetic moments calculated by OOMMF for $\operatorname{SmCo}(10 \mathrm{~nm}) / \mathrm{Fe}(20 \mathrm{~nm}) / \mathrm{SmCo}$ $(10 \mathrm{~nm})$, where the six states corresponds to the six marked points in the hysteresis loops shown in Fig. 2. The demonstrated moments locate at the middle plane of the soft layer, which respond to the applied field fast. (a) $H=14$ kOe, the magnetizations orient roughly at the applied field direction before the nucleation; (b) $H=-1 \mathrm{kOe}$, the magnetizations at the corners deviate more than $30^{\circ}$ away from the previous saturation state at the nucleation point; (c) $H=-3 \mathrm{kOe}$, the magnetization deviations at the top and bottom edges enhance after the nucleation; (d) $H=-6 \mathrm{kOe}$, where the change of the magnetizations at the edges lag behind (e) $H=-8.6 \mathrm{kOe}$, right at the coercive point; (f) $H=-13 \mathrm{kOe}$, magnetic reversal completed. The adopted ratio is 1:5 for presentation, i.e., one displayed magnetic moment at the figure stands for $5 \times 5$ calculated moments.

the corners of the film deviates slightly from the applied field direction as shown in Fig. 4(a). However, most magnetizations still orient roughly at the applied field direction, demonstrating a state before the nucleation. The 
corresponding average magnetization of the system is 113 $\mathrm{emu} / \mathrm{cc}$, which is roughly the saturation magnetization of the system, as shown in Fig. 2. When the field decreases to $1 \mathrm{kOe}$ (Fig. 4(b)), the magnetizations at the corners deviate more than $30^{\circ}$ away from the previous saturation direction, whereas those at the center still orient at the saturation direction. This field corresponds to the nucleation field of the system. Such deviations will enhance and spread nearby through the exchange interaction upon the further decrease of the applied field. As the field decreases to $-3 \mathrm{kOe}$, obvious deviation can be observed at the top and bottom edges as shown in Fig. 4(c). In particular, the magnetizations at the corners deviate roughly $50^{\circ}$ away from the the previous saturation direction already, whereas those at the center still orient at the previous saturation direction. As a result, the corresponding macroscopic magnetization is $107 \mathrm{emu} / \mathrm{cc}$. Further decrease of the applied field will lead to the rotation of the magnetizations at the center. At $H=-6 \mathrm{kOe}$ (Fig. 4(d)), almost all magnetizations within this film plane are perpendicular to the previous saturation direction Note that at this point, the response of the magnetizations at the edges with the applied field lag behind those at the centers, which have a deviation of roughly $60^{\circ}$ only. This state is still some distance away from the coercivity of the system, as the magnetizations in the hard layer are still at the previous saturation state. The corresponding average magnetization is $512 \mathrm{emu} / \mathrm{cc}$, as displayed in Fig. 2. The system will reach the coercive point at $H=-8.6 \mathrm{kOe}$ (Fig. 4(e)), where all the magnetizations in the middle plane of the soft layer orient at $120^{\circ}$ away from the previous saturation direction. Further decrease of the applied field will lead to the saturation of the system. At Fig. 4(f), the trilayer saturates at $H=-13 \mathrm{kOe}$.

\section{Angular Distribution at the Direction Perpendicular to the Film Plane and the Reversal Processes of Magnetization}

The above magnetic moment distribution at the film plane can display nucleation modes very well, which starts at the surface of the soft layer. However, the important magnetic reversal processes through domain wall motion is not revealed. To do this, the angular distribution at the $z$ direction should be discussed.

Fig. 5 shows the angular distributions of the magnetization at the thickness direction for the $\operatorname{SmCo}(10 \mathrm{~nm}) /$ $\mathrm{Fe}(5 \mathrm{~nm}) / \mathrm{SmCo}(10 \mathrm{~nm})$ trilayers based on $1 \mathrm{D}$ and $3 \mathrm{D}$ models. For the 1D calculation, the angular distribution at the $z$ direction could be obtained by minimizing the energy density expressed in Eq. (4), which is coherent at

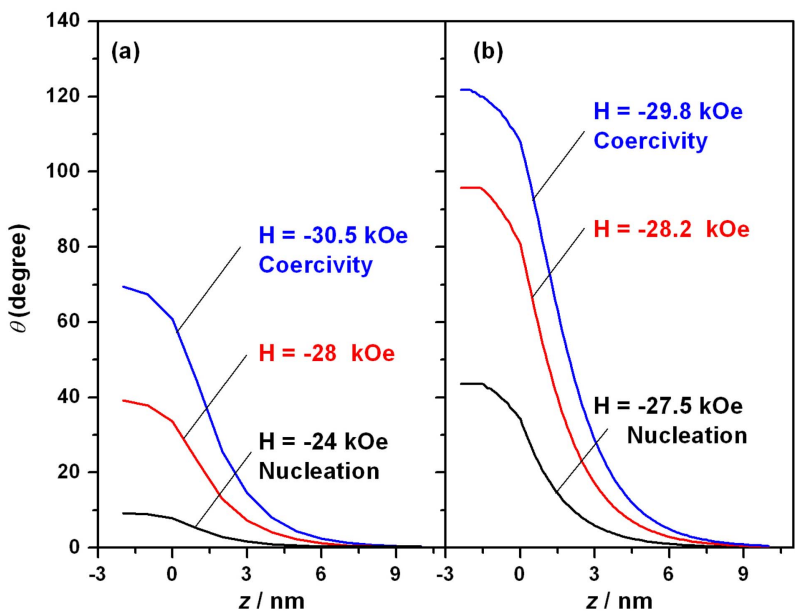

Fig. 5. (Color online) Calculated spatial distribution of magnetic orientation $q$ in the thickness direction at various applied fields for the $\operatorname{SmCo}(10 \mathrm{~nm}) / \mathrm{Fe}(5 \mathrm{~nm}) / \mathrm{SmCo}(10 \mathrm{~nm})$ trilayers (a) based on the 3D simulation and (b) based on the 1D model. Due to the symmetry, only magnetization distributions in the half of the trilayers have been drawn and shown.

$x-y$ plane. For the 3D calculation, the angular distribution is obtained by averaging the component of all the magnetic moments in the applied field direction with a certain value of $z$. Due to the symmetry, only magnetization distributions in the half of the trilayers has been drawn and shown.

According to the 3D calculation, a small deviation of the magnetization from the previous saturation state occurs at $H=-24 \mathrm{kOe}$, which is called nucleation according to Brown. At this field, a $9.2^{\circ}$ infant domain wall has been formed with $\theta^{h}=0^{\circ}, \theta^{0}=7.8^{\circ}$ and $\theta^{s}=9.2^{\circ}$. Only about $15 \%$ of the domain wall is within the soft phase due to the very thin soft layer. The angular increase of the magnetization can be observed as the applied field decreases. Exception is observed at the surface of hard phase, where $\theta^{h}$ keeps a constant of $0^{\circ}$ from nucleation to pinning. Due to the large thickness of the hard phase, the angle at the surface of the hard phase $\theta^{h}$ obeys the Stoner-Wohlfarth (SW) model [30], where $\theta^{h}=0^{\circ}$ before the magnetic reversal and $\theta^{h}=180^{\circ}$ after the reversal. In the meantime, the domain wall is pushed toward the hard phase gradually. When $H=-28 \mathrm{kOe}, \theta^{0}$ and $\theta^{5}$ increase to $33.7^{\circ}$ and $=39.2^{\circ}$ respectively, where $86 \%$ of the domain wall locates at the hard phase. When the applied field decreases to the coercivity $(H=-30.51 \mathrm{kOe}), \theta^{0}$ and $\theta^{\mathrm{s}}$ increase to $60.9^{\circ}$ and $69.5^{\circ}$ respectively.

The 1D calculation gives similar angular distributions, where the nucleation occurs at a more negative applied field $(H=-27.5 \mathrm{kOe})$. At the nucleation point, a $43.5^{\circ}$ domain has been formed in the trilayers, with $21 \%$ 
located at the soft phase. The corresponding $\theta^{0}$ and $\theta^{s}$ are $34.4^{\circ}$ and $43.5^{\circ}$ respectively. A slight further decrease of the field will lead to a large increase of both $\theta^{0}$ and $\theta^{\text {s. In }}$ the meantime, the domain wall moves toward the hard phase gradually. At $H=-28.2 \mathrm{kOe}, \theta^{0}$ and $\theta^{s}$ increase to $80.8^{\circ}$ and $95.7^{\circ}$ respectively, where roughly $85 \%$ of the domain wall is at the hard phase. At the coercivity (-29.82 $\mathrm{kOe}), \theta^{s}$ and $\theta^{0}$ increase to $121.7^{\circ}$ and $107.9^{\circ}$ respectively, where only $11 \%$ of the domain wall is in the soft phase. Further decrease of the applied field leads to the irreversible reversal of the magnetizations of the whole system.

Although the results of the $1 \mathrm{D}$ and $3 \mathrm{D}$ are similar, there are some differences between them. The angles according to the $3 \mathrm{D}$ calculations are systematically smaller than those based on the 1D calculations. In addition, the motion of the domain walls from the soft to the hard phase is more evident based on the 1D calculation, where the percentage of the domain wall within the hard phase increases from $79 \%$ at the nucleation to $89 \%$ at the coercivity. More importantly, the nucleation process based on the $3 \mathrm{D}$ calculations is much slower, leaving a large gap between nucleation and coercivity.

The angular distributions for other soft layer thicknesses have also been calculated and compared. Fig. 6 shows the calculated angular distributions for SmCo (10 $\mathrm{nm}) / \mathrm{Fe}(10 \mathrm{~nm}) / \mathrm{SmCo}(10 \mathrm{~nm})$ trilayers based on the two methods, which are similar to those for $L^{s}=5 \mathrm{~nm}$. Noticeably, the angles according to the 3D calculations are systematically smaller than those based on the 1D

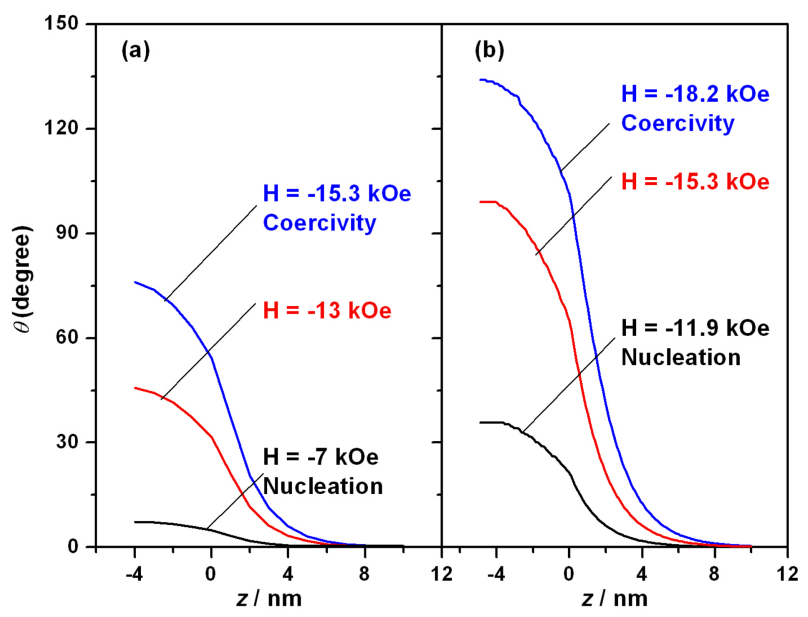

Fig. 6. (Color online) Calculated spatial distribution of magnetic orientation $q$ in the thickness direction at various applied fields for $\mathrm{SmCo}(10 \mathrm{~nm}) / \mathrm{Fe}(10 \mathrm{~nm}) / \mathrm{SmCo}(10 \mathrm{~nm})$ trilayers. (a) Calculated by the 3D simulation and (b) calculated by the 1D model. Due to the symmetry, only magnetization distributions in the half of the trilayers has been drawn and shown. calculations. Moreover, the motion of the domain walls from the soft to the hard phase is evidenced in the 1D results, where the percentage of the domain wall within the hard phase rises from $60 \%$ at the nucleation to $76 \%$ at the coercivity.

Compared with the case with $L^{s}=5 \mathrm{~nm}$, the percentage of the domain wall within the soft phase is larger, accompanied by the drop of both nucleation and coercive fields. According to the 3D simulation, a domain wall of $7.4^{\circ}$ forms in the trilayers at the nucleation point with $68 \%$ in the hard phase. The angle of the magnetization increases with the decreasing applied field. When $H$ decreases to $-13 \mathrm{kOe}$, the domain wall develops to $45.8^{\circ}$ and increase to $76.0^{\circ}$ at the coercive point, with $69 \%$ and $71 \%$ in the hard phase respectively.

In general, the $3 \mathrm{D}$ calculation yields better results than those given by the 1D calculation, as can be seen from Figs. 2 and 3 that the calculated coercivity and energy products based on the 3D calculation is generally smaller than that from the 1D calculation. For example, the calculated coercivity decreases from $15.3 \mathrm{kOe}$ to $8.6 \mathrm{kOe}$ as $L^{s}$ increase from $10 \mathrm{~nm}$ to $20 \mathrm{~nm}$ for $\operatorname{SmCo}(10 \mathrm{~nm})_{5} /$ $\mathrm{Fe} / \mathrm{SmCo}_{5}(10 \mathrm{~nm})$ trilayers according to the 3D calculation (see Table 1). On the other hand, the corresponding coercivity reduces from $18.2 \mathrm{kOe}$ to $8.6 \mathrm{kOe}$ according to the 3D calculation (see Table 1). Similar coercivity can be obtained for other $L^{h}$ as can be seen from Table 2 that the coercivity is not sensitive to the hard layer thickness. Whilst experimental coercivity drops from roughly 10 kOe to $7.5 \mathrm{kOe}$ achieved by Sawatzki et al. ${ }^{29}$ for $\mathrm{SmCo}_{5}$ $(25 \mathrm{~nm})_{5} / \mathrm{Fe}\left(L^{S}\right) / \mathrm{SmCo}_{5}(25 \mathrm{~nm})$ trilayers. Therefore, the

Table 1. Calculated magnetic properties for $\operatorname{SmCo}(10 \mathrm{~nm}) /$ $\mathrm{Fe}\left(L^{s}\right) / \mathrm{SmCo}(10 \mathrm{~nm})$ trilayers based on OOMMF (3D) and the simplified model (1D), where $H_{N}, H_{c}$ and $H_{P}$ denote the nucleation, coercive and pinning fields respectively, while $(B H)_{\max }$ and $M_{r}$ stand for the maximum energy product and the remanence.

\begin{tabular}{ccccccc}
\hline \hline $\begin{array}{c}L^{s} \\
(\mathrm{~nm})\end{array}$ & Method & $\begin{array}{c}H_{N} \\
(\mathrm{kOe})\end{array}$ & $\begin{array}{c}H_{C} \\
(\mathrm{kOe})\end{array}$ & $\begin{array}{c}H_{P} \\
(\mathrm{kOe})\end{array}$ & $\begin{array}{c}(B H)_{\max } \\
(\mathrm{MG} \mathrm{Oe})\end{array}$ & $\begin{array}{c}M_{r} \\
\left(\times 10^{3} \mathrm{emu} / \mathrm{cc}\right)\end{array}$ \\
\hline \multirow{2}{*}{2} & 1D & 67.3 & 67.3 & 67.3 & 16.9 & 0.65 \\
& 3D & 62.7 & 63.5 & 63.5 & 16.9 & 0.65 \\
\hline \multirow{2}{*}{5} & 1D & 27.5 & 29.8 & 29.8 & 24.0 & 0.78 \\
& 3D & 24.0 & 30.5 & 30.5 & 24.0 & 0.78 \\
\hline \multirow{2}{*}{10} & 1D & 11.9 & 18.2 & 18.4 & 34.4 & 0.93 \\
& 3D & 7.0 & 15.3 & 15.3 & 34.2 & 0.93 \\
\hline \multirow{2}{*}{15} & 1D & 6.9 & 12.5 & 15.4 & 42.9 & 1.04 \\
& 3D & 1.5 & 12.0 & 12.0 & 38.6 & 1.04 \\
\hline \multirow{2}{*}{20} & 1D & 4.9 & 8.6 & 14.1 & 40.6 & 1.13 \\
& 3D & -1.0 & 8.6 & 11.1 & 36.3 & 1.13 \\
\hline
\end{tabular}


Table 2. Calculated magnetic properties for $\operatorname{SmCo}\left(L^{h}\right) /$ $\mathrm{Fe}\left(L^{s}\right) / \mathrm{SmCo}\left(L^{h}\right)$ trilayers based on OOMMF, where $H_{N}, H_{c}$ and $H_{P}$ denote the nucleation, coercive and pinning fields respectively, while $(B H)_{\max }$ and $M_{r}$ stand for the maximum energy product and the remanence.

\begin{tabular}{ccccccc}
\hline \hline $\begin{array}{c}L^{s} \\
(\mathrm{~nm})\end{array}$ & $L^{h}(\mathrm{~nm})$ & $\begin{array}{c}H_{N} \\
(\mathrm{kOe})\end{array}$ & $\begin{array}{c}H_{C} \\
(\mathrm{kOe})\end{array}$ & $\begin{array}{c}H_{P} \\
(\mathrm{kOe})\end{array}$ & $\begin{array}{c}(B H)_{\max } \\
(\mathrm{MG} \mathrm{Oe})\end{array}$ & $\begin{array}{c}M_{r} \\
\left(\times 10^{3} \mathrm{emu} / \mathrm{cc}\right)\end{array}$ \\
\hline \multirow{3}{*}{5} & 5 & 23.8 & 30.4 & 30.4 & 34.3 & 0.93 \\
& 15 & 24.1 & 30.6 & 30.6 & 19.9 & 0.71 \\
& 20 & 24.2 & 30.6 & 30.6 & 18.1 & 0.68 \\
\hline \multirow{4}{*}{10} & 5 & 6.8 & 15.2 & 15.2 & 49.6 & 1.12 \\
& 15 & 7.1 & 15.4 & 15.4 & 27.5 & 0.84 \\
& 20 & 7.1 & 15.4 & 15.4 & 23.9 & 0.78 \\
\hline \multirow{3}{*}{15} & 5 & 1.5 & 9.8 & 9.8 & 52.8 & 1.24 \\
& 15 & 1.5 & 12.0 & 12.0 & 31.8 & 0.93 \\
& 20 & 1.5 & 12.0 & 12.0 & 27.5 & 0.86 \\
\hline \multirow{2}{*}{20} & 5 & -1.1 & 6.0 & 6.0 & 46.7 & 1.30 \\
& 15 & -1.0 & 9.1 & 11.1 & 30.9 & 1.00 \\
& 20 & -1.0 & 11.1 & 11.1 & 27.3 & 0.92 \\
\hline
\end{tabular}

coercivity gap between the experiment and the theory is shortened according to the 3D calculation, which can include the local stray fields. Similar situation holds for the energy product gap However, the 3D calculation requires much more computational power than the 1D calculation so that the mesh size in the $3 \mathrm{D}$ calculation is larger than that for the 1D calculation. Therefore it is hard to say which model is better. We use both methods in this paper and compare the results to justify our methods.

\section{Critical Fields and Energy Products}

From above discussions, it becomes clear that the most important magnetic properties for hard/soft multilayers are critical fields, i.e., nucleation and coercive fields. Fig. 7 highlights the calculated critical fields based on OOMMF and the 1D model for $\mathrm{SmCo}(10 \mathrm{~nm}) / \mathrm{Fe}\left(L^{s}\right) / \mathrm{SmCo}(10 \mathrm{~nm})$ trilayers, as functions of the soft layer thickness. It can be seen that the two models yield similar critical fields. For small $L^{s}$, the coercivity equals the nucleation field, whereas $H_{c}$ is larger than $H_{N}$ for large $L s$. As the soft layer thickness increases, both nucleation and coercive fields decrease. In the meantime, the gap between the nucleation and coercivity rises. Similar trend has been obtained for other hard/soft bilayers [10, 15, 18]. Experimentally, Sawatzki et al. [24] found that the coercivity is equal to the nucleation field in $\operatorname{SmCo}_{5}(25 \mathrm{~nm}) / / \mathrm{Fe} /$ $\mathrm{SmCo}_{5}(25 \mathrm{~nm})$ trilayers for small $L^{s}$, whereas $H_{c}$ becomes to deviate from the nucleation field when the soft layer thickness is above a critical value of $8.8 \mathrm{~nm}$. The gap

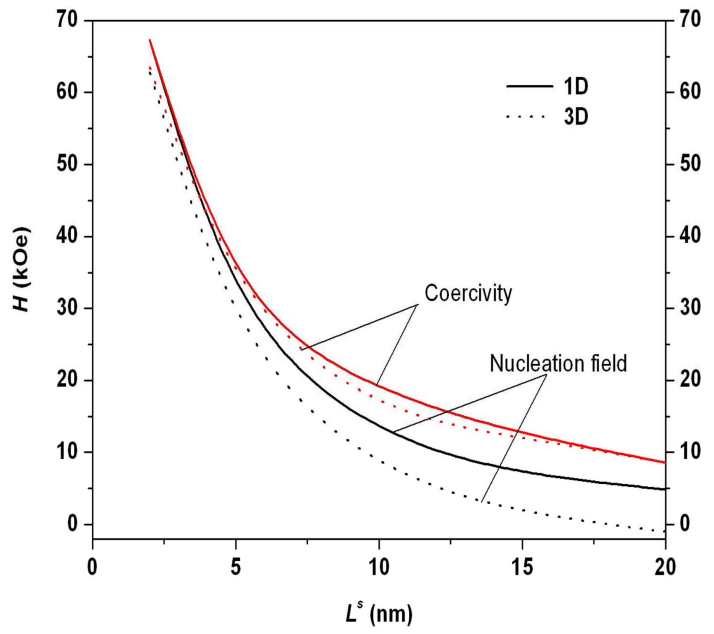

Fig. 7. (Color online) Calculated nucleation and coercive fields as functions of the soft layer thickness based on OOMMF (dotted lines) and 1D analytical model (Solid lines).

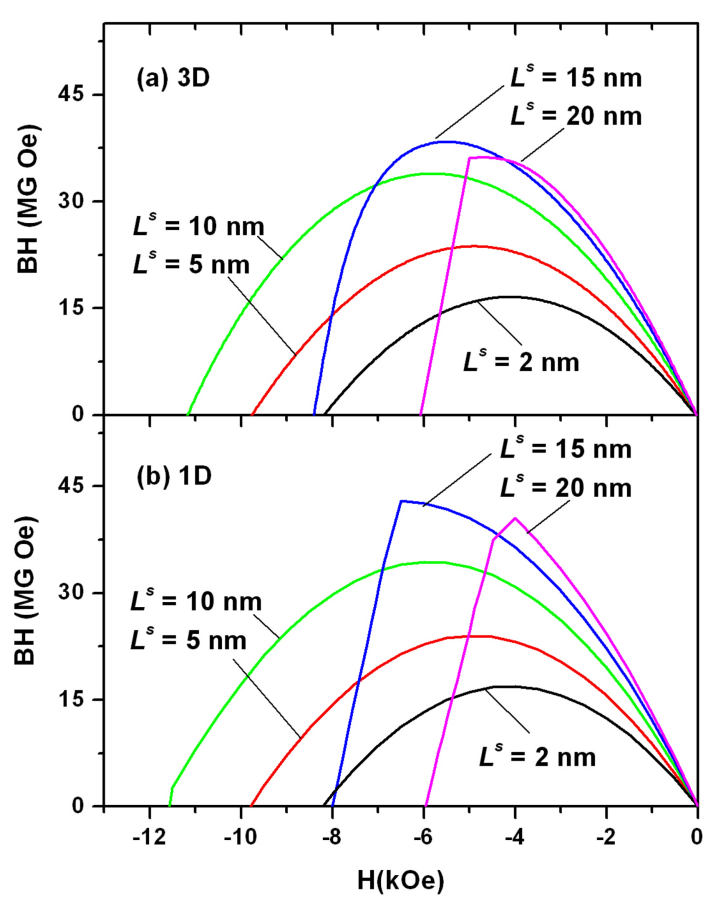

Fig. 8. (Color online) Calculated second-quadrant energy product $(B H)_{\max }$ for $\operatorname{SmCo}(10 \mathrm{~nm}) / \mathrm{Fe}\left(L^{s}\right) / \operatorname{SmCo}(10 \mathrm{~nm})$ trilayers. (a) Energy products based on OOMMF; (b) energy products based on the $1 \mathrm{D}$ calculation.

between $H_{c}$ and $H_{N}$ also increases with $L^{s}$. However, the experimental critical thickness is much larger than those shown in Fig. 8.

The calculated nucleation fields by the 3D simulation are systematically smaller than those based on the 1D calculation. The stray fields at the corners of the film plane are responsible for the difference. These demagneti- 
zation fields, taken into account by the OOMMF, facilitate the nucleation and lead to considerably lower nucleation fields. The stray fields will also have some influence on the calculated coercivities and energy products, which, however, is less significant compared with the former. For example, when $L^{s}=20 \mathrm{~nm}$, the calculated nucleation fields have opposite signs, whereas the difference for the coercivities are within $1 \%$. Therefore, the gap between the nucleation and the coercivity is larger according to the $3 \mathrm{D}$ calculation.

Fig. 8 shows the Calculated energy products for $\mathrm{SmCo}(10 \mathrm{~nm}) / \mathrm{Fe}\left(L^{s}\right) / \mathrm{SmCo}(10 \mathrm{~nm})$ trilayers based on 1D and $3 \mathrm{D}$ methods. Both models give similar energy products. In particular, the calculated maximum energy products are very close, justifying our calculation. For $L^{s}$ $\leq 10 \mathrm{~nm}$, both models give exactly the same $(B H)_{\max }$. Here the $(B H)_{\max }$ is achieved at the points before the nucleation takes place and thus not affected by the nucleation difference caused by the different models. For $L^{s} \geq 15 \mathrm{~nm}$, the $(B H)_{\max }$ based on the $3 \mathrm{D}$ calculation are smaller, due to the smaller nucleation fields obtained. However, the difference between the calculated $(B H)_{\max }$ according to the two models are still within $10 \%$.

Importantly, both models yield the largest $(B H)_{\max }$ at $L^{s}$ $=15 \mathrm{~nm}$, which is around 40 MGOe. These results agree well with available experimental data. Experimentally, Sawatzki et al. [24] obtained an energy product of 40 MGOe in $\mathrm{SmCo}_{5} / \mathrm{Fe} / \mathrm{SmCo}_{5}$ trilayers at $L^{s}=12.6 \mathrm{~nm}$.

The calculated magnetic properties for $\operatorname{SmCo}(10 \mathrm{~nm}) /$ $\mathrm{Fe}\left(L^{S}\right) / \mathrm{SmCo}(10 \mathrm{~nm})$ are summarized in Table 1. One can see that both models yield similar results. In particular, the calculated remanence, coercivity and energy products based on the two methods are very close, where the difference is within $10 \%$. As $L^{s}$ increases, the critical fields go up monotonically whereas the remanence falls down. Therefore, the energy products have a peak at $L^{s}=$ $15 \mathrm{~nm}$

On the other hand, the nucleation and pinning fields based on OOMMF calculations are systematically smaller than those based on the 1D model. The difference enlarges as $L^{s}$ increases. When $L^{s}=2 \mathrm{~nm}$, both differences for the nucleation and pinning fields are within $10 \%$. When $L^{s}=$ $20 \mathrm{~nm}$, however, the pinning field difference is as large as $30 \%$, while the nucleation fields have opposite signs. It should be addressed that although the nucleation field difference is significant at large $L^{s}$, it does not affect much on the energy products or the hysteresis loops. As has been mentioned above, the nucleation based on the $3 \mathrm{D}$ is a slow and smooth process. The magnetizations do not change much after the nucleation. The small nucleation fields compensated by the slow process from the nucleation to pinning, leading to roughly the same coercivity as that based on the 1D model. The coercivity mechanism is pure pinning for small $L^{s}$ according to the OOMMF calculation. It changes to a hybrid one at large $L^{s}$, where the coercivity is in between the nucleation and pinning fields. The coercivity mechanism based on the 1D model is more complicate. The coercivity equals both nucleation and pinning fields at small $L^{s}$. Such a mechanism changes to a pure pinning one at intermediate $L^{s}$ and finally to a hybrid one at large $L^{s}$.

In the particular case for $L^{s}=15 \mathrm{~nm}$ (see Table 1), in both models the $H_{N}$ are quite different. However, the coercivities is very similar as the coercivity is not determined by the nucleation field in this system. Rather, it is completely determined by the pinning field according to the $3 \mathrm{D}$ calculations. In the $1 \mathrm{D}$ calculation, the situation is slightly different; $H_{c}$ is in between the nucleation and the pinning fields but is more close to the pinning field. Further, the two models suggest similar $M_{r}$ and $H_{c}$, which are important reference properties for the largest energy product. Nevertheless, the $(B H)_{\max }$ values are more different as $(B H)_{\max }$ is not determined by these two values completely. $(B H)_{\max }$ is also closely related to the shape of the hysteresis loop in the second quadrant. In particular, the nucleation field based on the 3D model is much smaller than that according to the 1D model, leading to a smaller $(B H)_{\max }$.

The calculated magnetic properties corresponding to Fig. 3 for $\operatorname{SmCo}\left(L^{h}\right) / \mathrm{Fe}\left(L^{s}\right) / \operatorname{SmCo}\left(L^{h}\right)$ with other three values of $L^{h}$ are summarized in Table 2. One can see that the largest energy products also occur at $L^{s}=15 \mathrm{~nm}$ for all values of $L^{h}$. The highest $(B H)_{\max }$ rises from 27.5 MGOe to 52.8 MGOe as $L^{h}$ decreases from $20 \mathrm{~nm}$ to 5 $\mathrm{nm}$. Experimentaly, energy products of 40 MGOe has been achieved by Sawatzki et al. ${ }^{29}$ for $\operatorname{SmCo}_{5}(25 \mathrm{~nm})_{5} /$ $\mathrm{Fe}(12.6 \mathrm{~nm}) / \mathrm{SmCo}_{5}(25 \mathrm{~nm})$ trilayers. Both the value of $(B H)_{\max }$ and the soft layer thickness at which the highest energy products occur are in good agreement with the present model. On the other hand, the hard layer thickness at which the largest $(B H)_{\max }$ occurs is about $5 \mathrm{~nm}$, which is much smaller than the experimental one. Similar results have been obtained by other groups [4, 15-17]. For example, Skomski and Coey [4] recommend that the hard layer thickness to be less than $3 \mathrm{~nm}$ for the giant energy product to be realized. These deviations come from the fact that the effective anisotropy of the hard phase in the experiment might be less than the theoretical one [10]. Further, some possible distributions of easy axis orientations (although very small in the experiments) might affect the energy product and shift the optimum hard layer thickness to a higher value [11]. The formation of the Fe-Co [23, 
24] interlayer in the interface is the other contribution.

\section{Conclusions}

In this study, the properties of $\mathrm{Sm}-\mathrm{Co} / \alpha-\mathrm{Fe} / \mathrm{Sm}-\mathrm{Co}$ trilayers have been studied using a 3D micromagnetic software, OOMMF and compared carefully with those given by the $1 \mathrm{D}$ analytical model. It is found that the calculated hysteresis loops and angular distributions are consistent with each other, ensuring the reliability of our calculations. In particular, both nucleation and coercive fields decrease as the soft layer thickness increases. The difference for the remanence, coercivity and maximum energy products based on different models are less than $10 \%$. The calculated largest $(B H)_{\max }$ is around $50 \mathrm{MGOe}$, achieved at $L^{s}=15$ $\mathrm{nm}\left(L^{h}=5 \mathrm{~nm}\right)$, which is consistent with available experimental data.

The calculated nucleation and pinning fields based on OOMMF are systematically smaller than those according to the 1D model, where the difference increases as $L^{s}$ increases. These differences are due to the large stray fields acting on the magnetic moments at the corners of the film planes, which is ignored in the 1D calculation.

\section{Acknowledgement}

This work was supported by the National Natural Science Foundation of China (Grant No. 11074179, 11272070, 10747007), National 863 project of China (No. 2012AA040912), the Construction Plan for Scientific Research Innovation Teams of Universities in Sichuan Province (No. 12TD008).

\section{References}

[1] E. F. Kneller and R. Hawig, IEEE Trans. Magn. 27, 3588 (1991).

[2] E. E. Fullerton, J. S. Jiang, M. Grimsditch, C. H. Sowers, and S. D. Bader, Phys. Rev. B 58, 12193 (1998).

[3] M. Shindo, M. Ishizone, A. Sakuma, H. Kato, and T. Miyazaki, J. Appl. Phys. 81, 4444 (1997).

[4] R. Skomski and J. M. D. Coey, Phys. Rev. B 48, 15812 (1993).

[5] S. Sun, C. B. Murray, D. Weller, L. Folks, and A. Moser, Science 2871989 (2000).

[6] H. Zeng, J. Li, J. P. Liu, Z. L. Wang, and S. Sun, Nature 420395 (2002).

[7] D. J. Sellmyer, Nature 420, 374 (2002).
[8] B. Balasubramanian, R. Skomski, X.-Zh. Li, Sh. R. Valloppilly, J. E. Shield, G. C. Hadjipanayis, and D. J. Sellmyer, Nano Lett. 11, 1747 (2011).

[9] W. B. Cui, Y. K. Takahashi, and K. Hono, Adv. Mater. 24, 6530 (2012).

[10] G. P. Zhao and X. L. Wang, Phys. Rev. B 74, 012409 (2006).

[11] J. Xia, G. P. Zhao, H. W. Zhang, Z. H. Cheng, Y. P. Feng, J. Ding, and H. T. Yang, J. Appl. Phys. 112, 013918 (2012).

[12] E. Goto, N. Hayashi, T. Miyashita, and K. Nakagava, J. Appl. Phys. 36, 2951 (1965).

[13] T. Leineweber and H. Kronmüller, J. Magn. Magn. Mater. 176, 145 (1997).

[14] E. E. Fullerton, J. S. Jiang, and S. D. Bader, J. Magn. Magn. Mater. 200, 392 (1999).

[15] G. Asti, M. Solzi, M. Ghidini, and F. M. Neri, Phys. Rev. B 69, 174401 (2004).

[16] R. Pellicelli, M. Solzi, C. Pernechele, and M. Ghidini, Phys. Rev. B 83, 054434 (2011).

[17] R. Pellicelli, M. Solzi, V. Neu, K. Häfner, C. Pernechele, and A. M. Ghidini, Phys. Rev. B 81, 184430 (2010).

[18] G. P. Zhao, M. G. Zhao, H. S. Lim, Y. P. Feng, and C. K. Ong, Appl. Phys. Lett. 87, 162513 (2005).

[19] I. A. Al-Omari and D. J. Sellmyer, Phys. Rev. B 52, 3441 (1995).

[20] Z. H. Cheng, J. X. Zhang, and H. Kronmüller, Phys. Rev. B 68, 144417 (2003).

[21] V. Neu, K. Häfner, A. K. Patra, and L. Schultz, J. Phys. D: Appl. Phys. 39, 5116 (2006).

[22] X. B. Liu and Z. Altounian, J. Appl. Phys. 111, $07 B 526$ (2012).

[23] Y. Choi, J. S. Jiang, J. E. Pearson, S. D. Bader, J. J. Kavich, J. W. Freeland, and J. P. Liu, Appl. Phys. Lett. 91, 072509 (2007).

[24] S. Sawatzki, R. Heller, C. Mickel, M. Seifert, L. Schultz, and V. Neu, J. Appl. Phys. 109, 123922 (2011).

[25] M. J. Donahue and D. G. Porter, OOMMF user's guide, version 1.0. NISTIR 6376, National Institute of Standards and Technology, Gaithersburg, MD (1999).

[26] W. F. Brown, Jr., Rev. Mod. Phys. 17, 15 (1945).

[27] W. F. Brown, Micromagnetics (Interscience Publishers, New York, 1963).

[28] Y. Deng, G. P. Zhao, and H. W. Zhang, J. Magn. Magn. Mater 323, 535-538 (2011).

[29] G. Asti, M. Ghidini, R. Pellicelli, C. Pernechele, and M. Solzi, Phys. Rev. B 73, 094406 (2006).

[30] E. C. Stoner and E. P. Wohlfarth, Phil. Trans. R. Soc. 240, 599 (1948). 